\section{P2-S8.05 USE OF CELLULAR TELEPHONES IN A STUDY OF HIGH-RISK PATIENTS}

doi:10.1136/sextrans-2011-050108.379

D Craig, D Hensel, J D Fortenberry, J Harezlak, K Jones. School of Medicine, Indiana University, Indianapolis, USA

Objectives To describe interest, uptake, advantages and problems associated with the use of cellular phones in a study of incident STDs in a high-risk population.

Methods Study participants (SP) (N=357; 18-29 yrs; 91\% African American; 19.9\% incident STD at enrolment: Chlamydia, Gonorrhoea and Trichomoniasis) were enrolled in a 12-week study involving weekly STD sampling and three times daily electronic diary submission via cellular phone. SPs received 3 months of free unlimited calling, texting and internet browsing as part of study and an option to retain the phone at study conclusion.

Results Eligible SPs, the majority of whom were self-referred, were placed on a waiting list (volume range: $50-150$ people) until space in the study was available (avg time: 4 months). About $50 \%$ of persons on the waiting list were not enrolled; common barriers included invalid contact information and no call back. Advantages to SP cell phone use included: paperless data collection (SPs who completed the study [N=261] submitted $89.99 \%$ of expected diaries), facilitated communication between SP and study staff, free cell minutes/texting and internet, and the option to keep phone at study conclusion (about 80\%) as incentives to SPs. Challenges with cell phone use included issues with actual use (most common: failing to charge phone or poor care of phone), technical problems (most common: equipment failure [11.2\%] and connectivity problems $[21.1 \%]$ ), theft (about 10\%) and equipment breakage/damage $[14.8 \%])$. Once SP reported equipment issues, the time to replacement was short (average: 2 days).

Conclusion The data support the feasibility of using cellular phones in a high-risk population. Participants were not recruited for the waiting list, but self-referred. SPs were able to complete and submit surveys correctly and work with technical issues encountered.

\section{P2-S8.06 USING ELECTRONIC DIARIES PROSPECTIVELY TO CAPTURE STI-RELATED PHENOMENOLOGY}

\section{doi:10.1136/sextrans-2011-050108.380}

D Hensel, J D Fortenberry, J Harezlak, K Jones, D Craig. School of Medicine, Indiana University, Indianapolis, USA

Objective To describe electronic diary study adherence, completion and sexual behaviour patterns among a high-risk sample clinically recruited for a prospective study of incident STI.

Method Participants ( $N=243$; $18-29$ yrs; 61\% female; 88\% African American) completed 12 -weeks of weekly STI testing and $3 x$ /day electronic diaries assessing individual and partner-specific affect, daily activities, substance use and sexual and contraceptive behaviour. We analysed event- and subject-level completion rates, and subjectlevel STI and behaviour response effects among subjects who had completed the study and who had not been incarcerated during study. Results In total, $82.3 \%(200 / 243)$ of participants individually submitted at least 250 diaries (252 expected) during the study; as a group, participants submitted $89.7 \%$ (54900/61236) of the total number diaries expected. The average number of diaries weekly submitted varied between 17.03 and 19.45 (21 expected). Total and weekly submissions were similar by gender, age and race. The overall median survey completion time was $1.77 \mathrm{~min}$ (1.68 $\mathrm{min}$ : if no partners; $3.00 \mathrm{~min}$ : if any partners). Among those with only one partner, median completion time when no sex occurred was $2.47 \mathrm{~min}$ and was $3.87 \mathrm{~min}$ when any sex occurred. About 35\% (85/ 243 ) of participants had an STI at some point in the study. Subjects reported the following sexual behaviours: vaginal sex (86.4\%: $210 /$ $243)$, anal sex (29.6\%: 72/243), giving oral sex (70.8\%: 172/243) and receiving oral sex (82.7\%: 201/243). Of those reporting vaginal sex, $60.4 \%(127 / 210)$ had only one partner and $23.3 \%$ (49/210) reported two partners. The median number of partner changes was one. Event level data suggest no response effects: sensitive behaviours, such as substance use or vaginal sex, exhibited similar stability in reports over time as compared to non-sexual activities such as eating or taking a walk.

Conclusion Data suggest excellent study protocol adherence and stable behaviour reporting among a high-risk sample. These findings demonstrate the feasibility of prospective, incident STI data collection methods, particularly in clinically recruited samples, which are often assumed to be non-compliant or unreliable around such methods.

\section{P2-S8.07 KEEPING PARTICIPANTS ON BOARD: OPTIMISING UPTAKE BY AUTOMATED RESPONDENT REMINDERS IN AN INTERNET-BASED CHLAMYDIA SCREENING IN THE NETHERLANDS}

doi:10.1136/sextrans-2011-050108.381

${ }^{1} \mathrm{R}$ Koekenbier, ${ }^{1} \mathrm{~N}$ Dokkum, ${ }^{2} \mathrm{E}$ Op de Coul, ${ }^{3} \mathrm{~J}$ van Bergen, ${ }^{4} \mathrm{E}$ Brouwers, ${ }^{1} \mathrm{H}$ Fennema, ${ }^{5} \mathrm{H}$ Götz, ${ }^{4} \mathrm{C}$ Hoebe, ${ }^{3} \mathrm{~L}$ Pars, ${ }^{5} \mathrm{~S}$ van Ravesteijn. ${ }^{1}$ Amsterdam Public Health Service, Amsterdam, Netherlands; ${ }^{2}$ RIVM, Netherlands; ${ }^{3}$ STI AIDS Netherlands, Netherlands; ${ }^{4}$ South-Limburg Public Health Service, Netherlands; ${ }^{5}$ Rotterdam Rijnmond Public Health Service, Netherlands

Background A register- and Internet-based chlamydia screening started in 2008 among 16-29 year olds in Amsterdam, Rotterdam and South-Limburg, aiming to reduce population prevalence of chlamydia infections. Automated respondent reminders by letter, email and SMS were used to encourage and optimise participation. The contribution of reminders on package request and sample return was examined in relation to characteristics of the target population. Methods On average, 280000 persons were annually invited by means of a personal letter. Individuals who did not respond to the invitation letter received a reminder letter after 1 month. Email- and SMS reminders were sent to individuals who requested a test kit and did not return a sample or did not check their test results online. We evaluated the effect of reminders on response and participation rates in two screening rounds. Logistic regression analyses were conducted to identify determinants of providing a GSM number, late response (requesting a test package after a reminder letter), and late participation (returning a sample after email/SMS reminders). Results The overall package request rate in the first round increased from $12 \%$ to $20 \%$ (see Abstract P2-S8.06 Figure 1) after the reminder letter (in round 2 from $8 \%$ to 14\%). The proportion of individuals returning a sample increased from $10 \%$ to $16 \%$ after email/SMS reminders (in round 2 from $7 \%$ to 11\%). Of all respondents, $99 \%$ provided an email address and $72 \%$ a GSM number. Factors associated with providing a GSM number were younger age (16-24 yrs), non-Dutch background, lower educational level, symptoms of an STI, and sexual risk behaviour in the past 6 months $(p<0.05)$. Determinants for late response (requesting a test package after reminder letter) were male gender, young age (16-19yrs), non-Dutch background, having a casual partner or $\geq 2$ sex partners in $\leq 6$ months. The email/SMS reminders resulted in more men returning a sample. Persons with a higher sexual risk more often returned their sample before these reminders.

Conclusions Respondents' willingness to provide an email address or GSM number for communication during the screening procedure was high. Factors associated with providing a GSM number were also related to chlamydia positivity. Automated respondents reminders by letter influenced response rates (package request) and resulted in reaching more people at higher risk. Email- and SMS reminders contributed substantially to participation rates (sample return). 